\title{
Author Index Volume 40 (2014)
}

The issue number is given in front of the pagination

Abbate, C., B. Arosio, D. Galimberti, P. Nicolini, L.R. Chiara, P.D. Rossi, E. Ferri, C. Gussago, M. Deriz, C. Fenoglio, M. Serpente, E. Scarpini and D. Mari, Phenotypic Variability associated with the C9ORF72 Hexanucleotide Repeat Expansion: A Sporadic Case of Frontotemporal Lobar Degeneration with Prodromal Hyposmia and Predominant Semantic Deficits (4) 849-855

Abbate, C., see Gussago, C. (1) 45-49

Abisambra, J.F. and S. Scheff, Brain Injury in the Context of Tauopathies (3) 495-518

Acion, L., see García-Casares, N. (2) 375-386

Agostinho, P., see Rodrigues, D.I. (4) 981-992

Ahmed, S., see Flanagan, E.C. (1) 33-36

Alameda, F., see Ramos-Fernández, E. (3) 643-657

Alcolea, D., see Ramos-Fernández, E. (3) 643-657

Alesiani, M., see Cavedo, E. (4) 941-952

Algarzae, N.K., see Rice, A.C. (2) 319-330

Alho, E.J.L., see Kilimann, I. (3) 687-700

Al-Sarraj, S., see Gerschütz, A. (1) 135-142

Altieri, F., see Bedse, G. (3) 701-712

Alvarez-Retuerto, A.I., see Ting, S.K.S. (2) 271-275

Amaro Jr., E., see Kilimann, I. (3) 687-700

Ames, D., see Brodaty, H. (1) 221-226

Ames, D., see Buckley, R.F. (3) 551-561

Ames, D., see Rembach, A. (1) 95-104

Anfossi, M., see Piaceri, I. (3) 679-685

Angeloni, F., see Cavedo, E. (4) 941-952

Antonell, A., see Balasa, M. (4) 919-927

Aparecida Bento dos Santos, G., see Kilimann, I. (3) 687-700

Aquino, D., see Cavedo, E. (4) 941-952

Ariza Corbo, M.J., see García-Casares, N. (2) 375-386

Arosio, B., see Abbate, C. (4) 849-855

Arosio, B., see Gussago, C. (1) 45-49

Arzberger, T., see Gerschütz, A. (1) 135-142

Ashford, J.W., see Rosen, A.C. (2) 233-235

Asthana, S., see Wharton, W. (2) 331-341

Auriacombe, S., see Delva, F. (1) 57-67

Avila, J., see Pérez, M. (1) 143-151

Babiloni, C., see Cavedo, E. (4) 941-952
Baglieri, A., see Cavedo, E. (4) 941-952

Bagnoli, S., see Piaceri, I. (3) 679-685

Balasa, M., R. Sánchez-Valle, A. Antonell, B. Bosch, J. Olives, L. Rami, M. Castellví, J.L. Molinuevo and A. Lladó, Usefulness of Biomarkers in the Diagnosis and Prognosis of Early-Onset Cognitive Impairment (4) 919-927

Ballan, G., see Delva, F. (1) 57-67

Bamji-Mirza, M., D. Callaghan, D. Najem, S. Shen, M.S. Hasim, Z. Yang and W. Zhang, Stimulation of Insulin Signaling and Inhibition of JNK-AP1 Activation Protect Cells from Amyloid- $\beta$-Induced Signaling Dysregulation and Inflammatory Response (1) 105-122

Banaschewski, T., see Khan, W. (1) 37-43

Banks, W.A., see Farr, S.A. (4) 1005-1016

Baratti, C.M., see Feld, M. (1) 69-82

Barber, R.C., see Cunningham, R.L. (3) 563-573

Barber, R.C., see Hall, J.R. (4) 887-896

Barker, G.J., see Khan, W. (1) 37-43

Bartl, J., see Gerschütz, A. (1) 135-142

Bastianello, S., see Cavedo, E. (4) 941-952

Bayer, A., M. Phillips, G. Porter, U. Leonards, A. Bompas and A. Tales, Abnormal Inhibition of Return in Mild Cognitive Impairment: Is it Specific to the Presence of Prodromal Dementia? (1) 177-189

Bedse, G., A. Romano, S. Cianci, A.M. Lavecchia, P. Lorenzo, M.R. Elphick, F.M. LaFerla, G. Vendemiale, C. Grillo, F. Altieri, T. Cassano and S. Gaetani, Altered Expression of the CB1 Cannabinoid Receptor in the Triple Transgenic Mouse Model of Alzheimer's Disease (3) 701-712

Béjot, Y., see Jacquin, A. (4) 1029-1038

Bellec, P., see Wu, L. (4) 993-1004

Benito-León, J., see Romero, J.P. (2) 465-473

Benke, T., see Pertl, M.-T. (3) 531-540

Bennett Jr., J.P., see Rice, A.C. (2) 319-330

Benzinger, T., see Ting, S.K.S. (2) 271-275

Bermejo-Pareja, F., see Romero, J.P. (2) 465-473 
Bernardi, L., see Piaceri, I. (3) 679-685

Berthier, M.L., see García-Casares, N. (2) 375-386

Bessi, V., see Bracco, L. (3) 737-742

Binquet, C., see Jacquin, A. (4) 1029-1038

Bisoffi, M., see Solberg, N.O. (1) 191-212

Black, D.M., see Wharton, W. (2) 331-341

Blake, M.G., see Feld, M. (1) 69-82

Boada, M., see Ramos-Fernández, E. (3) 643-657

Boccia, M., M.C. Silveri and C. Guariglia, VisuoPerceptive Priming in Alzheimer's Disease: Evidence for a Multi-Componential Implicit Memory System (2) 455-463

Boccia, M.M., see Feld, M. (1) 69-82

Bodner, T., see Pertl, M.-T. (3) 531-540

Bokde, A.L.W., see Khan, W. (1) 37-43

Bokde, A.L.W., see Kilimann, I. (3) 687-700

Bompas, A., see Bayer, A. (1) 177-189

Bonithon-Kopp, C., see Jacquin, A. (4) 1029-1038

Bosch, B., see Balasa, M. (4) 919-927

Bosch-Morató, M., see Ramos-Fernández, E. (3) 643657

Bose, D., see Perez, F.P. (1) 1-17

Bouls, D., see Khan, W. (1) 37-43

Bozzali, M., see Minati, L. (1) 213-220

Bracco, L., V. Bessi, S. Padiglioni, S. Marini and G. Pepeu, Do Cholinesterase Inhibitors Act Primarily on Attention Deficit? A Naturalistic Study in Alzheimer's Disease Patients (3) 737-742

Brady, C.I., see Solberg, N.O. (1) 191-212

Brandner, S., see Zimmermann, R. (4) 1055-1060

Bredesen, D.E., see Zhang, Q. (3) 605-617

Bredin, A., see Delva, F. (1) 57-67

Brinton, E.A., see Wharton, W. (2) 331-341

Brodaty, H., M.H. Connors, J. Xu, M. Woodward and D. Ames, Predictors of Institutionalization in Dementia: A Three Year Longitudinal Study (1) 221-226

Brouwer, W., see Kraijo, H. (3) 631-642

Brown, D.C., see Solberg, N.O. (1) 191-212

Bruni, A.C., see Piaceri, I. (3) 679-685

Bruzzone, M.G., see Cavedo, E. (4) 941-952

Büchel, C., see Khan, W. (1) 37-43

Buckley, R.F., M.M. Saling, M. Irish, D. Ames, C.C. Rowe, N.T. Lautenschlager, P. Maruff, S.L. Macaulay, R.N. Martins, C.L. Masters, S.R. Rainey-Smith, A. Rembach, G. Savage, C. Szoeke, Personal Memory Function in Mild Cognitive Impairment and Subjective Memory Complaints: Results from the Australian Imaging, Biomarkers, and Lifestyle (AIBL) Study of Ageing (3) 551-561
Budoff, M., see Wharton, W. (2) 331-341

Burnham, S.C., see Rembach, A. (1) 95-104

Bush, A.I., see Rembach, A. (1) 95-104

Bystryak, S., see Maltsev, A.V. (1) 19-32

Caan, M.W.A., see Schmand, B. (2) 409-418

Callaghan, D., see Bamji-Mirza, M. (1) 105-122

Caltagirone, C., see Minati, L. (1) 213-220

Campanelli, A., see Gharbiya, M. (4) 907-917

Cao, J., see Shi, Z. (2) 277-283

Carbonnell, F., see Wu, L. (4) 993-1004

Carducci, F., see Cavedo, E. (4) 941-952

Carlsson, C.M., see Wharton, W. (2) 331-341

Casati, M., see Gussago, C. (1) 45-49

Cassano, T., see Bedse, G. (3) 701-712

Castellví, M., see Balasa, M. (4) 919-927

Cavedo, E., A. Redolfi, F. Angeloni, C. Babiloni, R. Lizio, L. Chiapparini, M.G. Bruzzone, D. Aquino, U. Sabatini, M. Alesiani, A. Cherubini, E. Salvatore, A. Soricelli, F. Vernieri, F. Scrascia, E. Sinforiani, P. Chiarati, S. Bastianello, P. Montella, D. Corbo, G. Tedeschi, S. Marino, A. Baglieri, S. De Salvo, F. Carducci, C.C. Quattrocchi, M. Cobelli and G.B. Frisoni, The Italian Alzheimer's Disease Neuroimaging Initiative (I-ADNI): Validation of Structural MR Imaging (4) 941-952 Cedars, M.I., see Wharton, W. (2) 331-341

Cellar, J., see Steenland, K. (3) 587-594

Cercignani, M., see Minati, L. (1) 213-220

Chakraverty, S., see Ting, S.K.S. (2) 271-275

Chamberlin, R., see Solberg, N.O. (1) 191-212

Chan, D., see Minati, L. (1) 213-220

Checler, F., see Meckler, X. (1) 161-176

Chen, J., see Zhong, X. (4) 953-966

Chen, K., see Sun, X. (1) 123-134

Chen, S., see Yang, Y. (4) 929-939

Chen, X., see Zhong, X. (4) 953-966

Chen, Y., see Sun, X. (1) 123-134

Chen, Y.-D., see Zhang, J. (1) 153-159

Cheng, R., see Janicki, S.C. (1) 83-93

Cheng, Y., see Shi, Z. (2) 277-283

Cherubini, A., see Cavedo, E. (4) 941-952

Chiapparini, L., see Cavedo, E. (4) 941-952

Chiara, L.R., see Abbate, C. (4) 849-855

Chiarati, P., see Cavedo, E. (4) 941-952

Chin, J., see Cho, H. (2) 285-295

Cho, H., J.-H. Kim, C. Kim, B.S. Ye, H.J. Kim, C.W. Yoon, Y. Noh, G.H. Kim, Y.J. Kim, J.-H. Kim, C.-H. Kim, S.J. Kang, J. Chin, S.T. Kim, K.-H. Lee, D.L. Na, J.-K. Seong and S.W. Seo, Shape 
Changes of the Basal Ganglia and Thalamus in Alzheimer's Disease: A Three-Year Longitudinal Study (2) 285-295

Cho, H., see Noh, Y. (4) 877-886

Choe, Y.S., see Noh, Y. (4) 877-886

Cianci, S., see Bedse, G. (3) 701-712

Clark, L.N., see Janicki, S.C. (1) 83-93

Clementy, A., see Delva, F. (1) 57-67

Cobelli, M., see Cavedo, E. (4) 941-952

Colao, R., see Piaceri, I. (3) 679-685

Connors, M.H., see Brodaty, H. (1) 221-226

Conrod, P., see Khan, W. (1) 37-43

Coppola, G., see Ting, S.K.S. (2) 271-275

Corbo, D., see Cavedo, E. (4) 941-952

Cruciani, F., see Gharbiya, M. (4) 907-917

Cuadros, R., see Pérez, M. (1) 143-151

Cuello, A.C., see Hanzel, C.E. (3) 667-678

Cunha, R.A., see Rodrigues, D.I. (4) 981-992

Cunningham, R., see Hall, J.R. (4) 887-896

Cunningham, R.L., M. Singh, S.E. O’Bryant, J.R. Hall and R.C. Barber, Oxidative Stress, Testosterone, and Cognition among Caucasian and MexicanAmerican Men with and without Alzheimer's Disease (3) 563-573

Cupidi, C., see Piaceri, I. (3) 679-685

D'Alton, S., see George, D.R. (4) 1061-1062

D’Antonio, F., see Gharbiya, M. (4) 907-917

Dagher, A., see Wu, L. (4) 993-1004

Dalby, N.O., C. Volbracht, L. Helboe, P.H. Larsen, H.S. Jensen, J. Egebjerg and A.B. Elvang, Altered Function of Hippocampal CA1 Pyramidal Neurons in the rTg4510 Mouse Model of Tauopathy (2) 429-442

Dartigues, J.-F., see Delva, F. (1) 57-67

Daubail, B., see Jacquin, A. (4) 1029-1038

David, R., see Yakhia, M. (4) 869-875

de Boer, M.E., see van der Steen, J.T. (3) 743-757

de Leeuw, R., see Kraijo, H. (3) 631-642

De Lena, C., see Gharbiya, M. (4) 907-917

De Salvo, S., see Cavedo, E. (4) 941-952

de Vet, H.C.W., see van der Steen, J.T. (3) 743-757

De Vico, U., see Gharbiya, M. (4) 907-917

Deck, L.M., see Solberg, N.O. (1) 191-212

Deckert, J., see Gerschütz, A. (1) 135-142

Del Campo, M. and C.E. Teunissen, Role of BRI2 in Dementia (3) 481-494

Delabrousse-Mayoux, J.-P., see Delva, F. (1) 57-67

Delazer, M., see Pertl, M.-T. (3) 531-540

Deliens, L., see van der Steen, J.T. (3) 743-757

Dell'Acqua, F., see Khan, W. (1) 37-43
Delva, F., S. Auriacombe, L. Letenneur, A. FoubertSamier, A. Bredin, A. Clementy, C. Latxague, E. Puymirat, G. Ballan, J.-P. Delabrousse-Mayoux, L. Glénisson, L. Mazat, U. Spampinato, M. Rainfray, F. Tison and J.-F. Dartigues, Natural History of Functional Decline in Alzheimer's Disease: A Systematic Review (1) 57-67

Deriz, M., see Abbate, C. (4) 849-855

Descamps, O., see Zhang, Q. (3) 605-617

Desobry, K., see Grammas, P. (3) 619-630

Dias, I.H.K., M.C. Polidori, L. Li, D. Weber, W. Stahl, G. Nelles, T. Grune and H.R. Griffiths, Plasma Levels of HDL and Carotenoids are Lower in Dementia Patients with Vascular Comorbidities (2) $399-408$

Dobson, R., see Khan, W. (1) 37-43

Doecke, J.D., see Rembach, A. (1) 95-104

Dowling, N.M., see Wharton, W. (2) 331-341

Dursun, E., see Gezen-Ak, D. (2) 257-269

Edwards, M., see Hall, J.R. (4) 887-896

Egebjerg, J., see Dalby, N.O. (2) 429-442

Ellis, K.A., see Rembach, A. (1) 95-104

Elosúa, R., see Ramos-Fernández, E. (3) 643-657

Elphick, M.R., see Bedse, G. (3) 701-712

Elvang, A.B., see Dalby, N.O. (2) 429-442

Emídio da Silva, R., see Kilimann, I. (3) 687-700

Eraso-Pichot, A., see Ramos-Fernández, E. (3) 643-657

Erickson, M.A., see Farr, S.A. (4) 1005-1016

Eriksdotter, M., see Hanzel, C.E. (3) 667-678

Eustace, J., see O'Caoimh, R. (3) 595-603

Evola, M., see Grammas, P. (3) 619-630

Ewers, M., see Noh, Y. (4) 877-886

Eyjolfsdottir, H., see Hanzel, C.E. (3) 667-678

Fagan, A., see Ting, S.K.S. (2) 271-275

Fallgatter, A.J., see Gerschütz, A. (1) 135-142

Fang, Y., see Zhong, X. (4) 953-966

Farr, S.A., M.A.Erickson, M.L. Niehoff, W.A. Banks and J.E. Morley, Central and Peripheral Administration of Antisense Oligonucleotide Targeting Amyloid-b Protein Precursor Improves Learning and Memory and Reduces Neuroinflammatory Cytokines in Tg2576 (AbPPswe) Mice (4) 1005-1016

Feld, M., M.C. Krawczyk, M.S. Fustiñana, M.G. Blake, C.M. Baratti, A. Romano and M.M. Boccia, Decrease of ERK/MAPK Overactivation in Prefrontal Cortex Reverses Early Memory Deficit in a Mouse Model of Alzheimer's Disease (1) 6982 
Fellgiebel, A., see Kilimann, I. (3) 687-700

Feng, W., see Shi, Z. (2) 277-283

Fenoglio, C., see Abbate, C. (4) 849-855

Fernandes dos Reis, M.E., see Rosa, M.I. (2) 443-454

Fernandes, B., see Rosa, M.I. (2) 443-454

Fernández-Busquets, X., see Ramos-Fernández, E. (3) 643-657

Ferrer, I., see Gerschütz, A. (1) 135-142

Ferri, E., see Abbate, C. (4) 849-855

Ferri, E., see Gussago, C. (1) 45-49

Filippi, M., see Kilimann, I. (3) 687-700

Fischer, M., see Gerschütz, A. (1) 135-142

Flanagan, E.C., S. Tu, S. Ahmed, J.R. Hodges and M. Hornberger, Memory and Orientation in the Logopenic and Nonfluent Subtypes of Primary Progressive Aphasia (1) 33-36

Flor, H., see Khan, W. (1) 37-43

Fonov, V., see Wu, L. (4) 993-1004

Fortea, J., see Ramos-Fernández, E. (3) 643-657

Foubert-Samier, A., see Delva, F. (1) 57-67

Friedman, L., see Yakhia, M. (4) 869-875

Frisoni, G.B., see Cavedo, E. (4) 941-952

Frisoni, G.B., see Kilimann, I. (3) 687-700

Frouin, V., see Khan, W. (1) 37-43

Funata, N., see Homma, T. (1) 51-55

Fustiñana, M.S., see Feld, M. (1) 69-82

Gaetani, S., see Bedse, G. (3) 701-712

Galimberti, D., see Abbate, C. (4) 849-855

Gallinat, J., see Khan, W. (1) 37-43

Gallo, M., see Piaceri, I. (3) 679-685

Galzitskaya, O.V., see Maltsev, A.V. (1) 19-32

Gao, H.-Q., see Zhang, J. (1) 153-159

Gao, Y., see O'Caoimh, R. (3) 595-603

Garavan, H., see Khan, W. (1) 37-43

García, C., see Pérez, M. (1) 143-151

García-Arnés, J.A., see García-Casares, N. (2) 375-386

García-Casares, N., M.L. Berthier, R.E. Jorge, P. Gonzalez-Alegre, A. Gutiérrez Cardo, J. Rioja Villodres, L. Acion, M.J. Ariza Corbo, A. Nabrozidis, J.A. García-Arnés and P. GonzálezSantos, Structural and Functional Brain Changes in Middle-Aged Type 2 Diabetic Patients: A Cross-Sectional Study (2) 375-386

Garwood, M., see Solberg, N.O. (1) 191-212

Gauthier, S., see Wu, L. (4) 993-1004

Gay, D., see Grammas, P. (3) 619-630

George, D.R. and S. D'Alton, Review of "You're Looking At Me Like I Live Here And I Don't" (4) 1061-1062
Gerschütz, A., H. Heinsen, E. Grünblatt, A.K. Wagner, J. Bartl, C. Meissner, A.J. Fallgatter, S. Al-Sarraj, C. Troakes, I. Ferrer, T. Arzberger, J. Deckert, P. Riederer, M. Fischer, T. Tatschner and C.M. Monoranu, Neuron-Specific Alterations in Signal Transduction Pathways associated with Alzheimer's Disease (1) 135-142

Gezen-Ak, D., S. Yilmazer and E. Dursun, Why Vitamin $\mathrm{D}$ in Alzheimer's Disease? The Hypothesis (2) 257-269

Gharbiya, M., A. Trebbastoni, F. Parisi, S. Manganiello, F. Cruciani, F. D’Antonio, U. De Vico, L. Imbriano, A. Campanelli and C. De Lena, Choroidal Thinning as a New Finding in Alzheimer's Disease: Evidence from Enhanced Depth Imaging Spectral Domain Optical Coherence Tomography (4) $907-917$

Giampietro, V., see Khan, W. (1) 37-43

Ginestet, C., see Khan, W. (1) 37-43

Giroud, M., see Jacquin, A. (4) 1029-1038

Gleason, C.E., see Wharton, W. (2) 331-341

Glénisson, L., see Delva, F. (1) 57-67

Goate, A., see Ting, S.K.S. (2) 271-275

Goldstein, F., see Steenland, K. (3) 587-594

Gong, C.-X., see Liu, X. (2) 419-427

Gonzalez-Alegre, P., see García-Casares, N. (2) 375 386

González-Santos, P., see García-Casares, N. (2) $375-$ 386

Gorostiza, O., see Zhang, Q. (3) 605-617

Gowland, P., see Khan, W. (1) 37-43

Grammas, P., J. Martinez, A. Sanchez, X. Yin, J. Riley, D. Gay, K. Desobry, D. Tripathy, J. Luo, M. Evola and A. Young, A New Paradigm for the Treatment of Alzheimer's Disease: Targeting Vascular Activation (3) 619-630

Graule-Petot, A., see Jacquin, A. (4) 1029-1038

Gray, N.E., J. Morré, J. Kelley, C.S. Maier, J.F. Stevens, J.F. Quinn and A. Soumyanath, Caffeoylquinic Acids in Centella asiatica Protect against Amyloid- $\beta$ Toxicity (2) 359-373

Griffiths, H.R., see Dias, I.H.K. (2) 399-408

Grillo, C., see Bedse, G. (3) 701-712

Grinberg, L., see Kilimann, I. (3) 687-700

Groblewska, M., see Mroczko, B. (2) 351-357

Grothe, M., see Kilimann, I. (3) 687-700

Grünblatt, E., see Gerschütz, A. (1) 135-142

Grune, T., see Dias, I.H.K. (2) 399-408

Gualandris, F., see Gussago, C. (1) 45-49

Guariglia, C., see Boccia, M. (2) 455-463 
Guivernau, B., see Ramos-Fernández, E. (3) 643-657

Gussago, C., B. Arosio, M. Casati, E. Ferri, F. Gualandris, E. Tedone, P. Nicolini, P.D. Rossi, C. Abbate and D. Mari, Different Adenosine $A_{2 A}$ Receptor Expression in Peripheral Cells from Elderly Patients with Vascular Dementia and Alzheimer's Disease (1) 45-49

Gussago, C., see Abbate, C. (4) 849-855

Gutierres, J., see Rodrigues, D.I. (4) 981-992

Gutiérrez Cardo, A., see García-Casares, N. (2) 375386

Guyatt, G., see O’Caoimh, R. (3) 595-603

Hall, J.R., A.R. Wiechmann, L.A. Johnson, M. Edwards, R.C. Barber, R. Cunningham, M. Singh and S.E. O'Bryant, The Impact of APOE Status on Relationship of Biomarkers of Vascular Risk and Systemic Inflammation to Neuropsychiatric Symptoms in Alzheimer's Disease (4) 887-896

Hall, J.R., see Cunningham, R.L. (3) 563-573

Hall, S., see Nielsen, H.M. (2) 343-350

Hallie-Heierman, M., see van der Steen, J.T. (3) 743757

Hampel, H., see Kilimann, I. (3) 687-700

Hannequin, D., see Troussière, A.C. (4) 857-861

Hansson, O., see Nielsen, H.M. (2) 343-350

Hanzel, C.E., M.F. Iulita, H. Eyjolfsdottir, E. Hjorth, M. Schultzberg, M. Eriksdotter and A.C. Cuello, Analysis of Matrix Metallo-Proteases and the Plasminogen System in Mild Cognitive Impairment and Alzheimer's Disease Cerebrospinal Fluid (3) 667-678

Harman, S.M., see Wharton, W. (2) 331-341

Hart, M.J., see Zhang, Q. (3) 605-617

Hasim, M.S., see Bamji-Mirza, M. (1) 105-122

He, R.-Q., see Qiang, M. (4) 1039-1053

He, Y., see Liu, X. (2) 387-397

Healy, L., see O'Caoimh, R. (3) 595-603

Hecimovic, S., see Ting, S.K.S. (2) $271-275$

Heidrich, J.E., see Solberg, N.O. (1) 191-212

Heinsen, H., see Gerschütz, A. (1) 135-142

Heinsen, H., see Kilimann, I. (3) 687-700

Heinz, A., see Khan, W. (1) 37-43

Helboe, L., see Dalby, N.O. (2) 429-442

Hernández, F., see Pérez, M. (1) 143-151

Hertogh, C.M.P.M., see van der Steen, J.T. (3) 743-757

Herukka, S.-K., see Yang, H. (3) 659-666

Hjorth, E., see Hanzel, C.E. (3) 667-678

Hodges, J.R., see Flanagan, E.C. (1) 33-36

Hodges, J.R., see Savage, S.A. (2) 309-317
Hodis, H.N., see Wharton, W. (2) 331-341

Homma, T., H. Takubo, K. Takahashi, S. Matsubara, M. Takahashi, N. Funata, Y. Mochizuki, T. Mizutani, T. Komori and T. Uchihara, Lateralized Cortical Involvement and Contralateral Parkinsonism without Basal Ganglia Involvement in Two Autopsy Cases of Corticobasal SyndromeAlzheimer's Disease (1) 51-55

Hornberger, M., see Flanagan, E.C. (1) 33-36

Hou, L., see Zhong, X. (4) 953-966

Hu, J., see Liu, X. (2) 419-427

$\mathrm{Hu}$, Y., J. Liang and S. Yu, High Prevalence of Diabetes Mellitus in a Five-Generation Chinese Family with Huntington's Disease (4) 863-868

Huang, G., see Zhong, X. (4) 953-966

Huang, P., see Sui, X. (3) 575-586

Huber, E., see Zimmermann, R. (4) 1055-1060

Hugon, J., see Troussière, A.C. (4) 857-861

Hutmacher, M.M., see Ito, K. (4) 967-979

ILL-Raga, G., see Ramos-Fernández, E. (3) 643-657

Imbriano, L., see Gharbiya, M. (4) 907-917

Irish, M., see Buckley, R.F. (3) 551-561

Ito, K. and M.M. Hutmacher, Predicting the Time to Clinically Worsening in Mild Cognitive Impairment Patients and its Utility in Clinical Trial Design by Modeling a Longitudinal Clinical Dementia Rating Sum of Boxes from the ADNI Database (4) 967-979

Ittermann, B., see Khan, W. (1) 37-43

Iulita, M.F., see Hanzel, C.E. (3) 667-678

Jacquin, A., C. Binquet, O. Rouaud, A. Graule-Petot, B. Daubail, G.-V. Osseby, C. Bonithon-Kopp, M. Giroud and Y. Béjot, Post-Stroke Cognitive Impairment: High Prevalence and Determining Factors in a Cohort of Mild Stroke (4) 1029-1038

Jagt, D.L.V., see Solberg, N.O. (1) 191-212

Jagt, T.A.V., see Solberg, N.O. (1) 191-212

Jakubowski, H., see Suszyńska-Zajczyk, J. (3) 713-726

Janicki, S.C., N. Park, R. Cheng, J.H. Lee, N. Schupf and L.N. Clark, Estrogen Receptor $\beta$ Variants Modify Risk for Alzheimer's Disease in a Multiethnic Female Cohort (1) 83-93

Jensen, H.S., see Dalby, N.O. (2) 429-442

Jeon, S., see Noh, Y. (4) 877-886

Jia, J., see Sun, X. (1) 123-134

Jia, J., see Wu, L. (4) 993-1004

Jiang, T., see Tan, L. (4) 1017-1027

John, V., see Zhang, Q. (3) 605-617 
Johnson, L.A., see Hall, J.R. (4) 887-896

Jones, R., see Miners, J.S. (3) 727-735

Jorge, R.E., see García-Casares, N. (2) 375-386

Jurado-Arjona, J., see Pérez, M. (1) 143-151

Kane, D.J., see Zhang, Q. (3) 605-617

Kang, D.R., see Noh, Y. (4) 877-886

Kang, S.J., see Cho, H. (2) 285-295

Karner, E., see Pertl, M.-T. (3) 531-540

Keeney, P.M., see Rice, A.C. (2) 319-330

Kehoe, P.G., see O'Caoimh, R. (3) 595-603

Kelley, J., see Gray, N.E. (2) 359-373

Kempuraj, D., see Xiong, Z. (2) 297-308

Kepe, V., see Ting, S.K.S. (2) 271-275

Kerins, D.M., see O'Caoimh, R. (3) 595-603

Khan, W., V. Giampietro, C. Ginestet, F. Dell'Acqua, D. Bouls, S. Newhouse, R. Dobson, T. Banaschewski, G.J. Barker, A.L.W. Bokde, C. Büchel, P. Conrod, H. Flor, V. Frouin, H. Garavan, P. Gowland, A. Heinz, B. Ittermann, H. Lemaître, F. Nees, T. Paus, Z. Pausova, M. Rietschel, M.N. Smolka, A. Ströhle, J. Gallinat, E. Westman, G. Schumann, S. Lovestone, A. Simmons, No Differences in Hippocampal Volume between Carriers and NonCarriers of the ApoE $\varepsilon 4$ and $\varepsilon 2$ Alleles in Young Healthy Adolescents (1) 37-43

Kilimann, I., M. Grothe, H. Heinsen, E.J.L. Alho, L. Grinberg, E. Amaro Jr., G. Aparecida Bento dos Santos, R. Emídio da Silva, A.J. Mitchell, G.B. Frisoni, A.L.W. Bokde, A. Fellgiebel, M. Filippi, H. Hampel, S. Klöppel and S.J. Teipel, Subregional Basal Forebrain Atrophy in Alzheimer's Disease: A Multicenter Study (3) 687-700

Kim, C., see Cho, H. (2) 285-295

Kim, C.-H., see Cho, H. (2) 285-295

Kim, C.S., see Noh, Y. (4) 877-886

Kim, G.H., see Cho, H. (2) 285-295

Kim, G.H., see Noh, Y. (4) 877-886

Kim, H.J., see Cho, H. (2) 285-295

Kim, H.J., see Noh, Y. (4) 877-886

Kim, J.-H., see Cho, H. (2) 285-295

Kim, J.-H., see Cho, H. (2) 285-295

Kim, J.-H., see Noh, Y. (4) 877-886

Kim, J.S., see Noh, Y. (4) 877-886

Kim, S.T., see Cho, H. (2) 285-295

Kim, S.T., see Noh, Y. (4) 877-886

Kim, Y.J., see Cho, H. (2) 285-295

Klöppel, S., see Kilimann, I. (3) 687-700

Komori, T., see Homma, T. (1) 51-55

König, A., see Yakhia, M. (4) 869-875

Koper, O.M., see Mroczko, B. (2) 351-357
Kornhuber, J., see Mroczko, B. (2) 351-357

Kornhuber, J., see Zimmermann, R. (4) 1055-1060

Kraijo, H., W. Brouwer, R. de Leeuw, G. Schrijvers and J. van Exel, The Perseverance Time of Informal Carers of Dementia Patients: Validation of a New Measure to Initiate Transition of Care at Home to Nursing Home Care (3) 631-642

Krantic, S. and A. Torriglia, Retina: Source of the Earliest Biomarkers for Alzheimer's Disease? (2) 237-243

Krawczyk, M.C., see Feld, M. (1) 69-82

Kulczyńska, A., see Mroczko, B. (2) 351-357

Ladd, A.C., see Rice, A.C. (2) 319-330

LaFerla, F.M., see Bedse, G. (3) 701-712

Lahiri, D.K., see Perez, F.P. (1) 1-17

Langa, E., see Pérez, M. (1) 143-151

Larsen, P.H., see Dalby, N.O. (2) 429-442

Latxague, C., see Delva, F. (1) 57-67

Lautenschlager, N.T., see Buckley, R.F. (3) 551-561

Lavecchia, A.M., see Bedse, G. (3) 701-712

Lee, J.H., see Janicki, S.C. (1) 83-93

Lee, J.-H., see Noh, Y. (4) 877-886

Lee, J.M., see Noh, Y. (4) 877-886

Lee, K.-H., see Cho, H. (2) 285-295

Lee, K.-H., see Noh, Y. (4) 877-886

Lelental, N., see Zimmermann, R. (4) 1055-1060

Lemaitre, H., see Khan, W. (1) 37-43

Leonards, U., see Bayer, A. (1) 177-189

Letenneur, L., see Delva, F. (1) 57-67

Lewczuk, P., see Mroczko, B. (2) 351-357

Lewczuk, P., see Zimmermann, R. (4) 1055-1060

Li, C., see Shi, Z. (2) 277-283

Li, C., see Yang, Y. (4) 929-939

Li, L., see Dias, I.H.K. (2) 399-408

Li, R.-J., see Zhang, J. (1) 153-159

Li, S., see Sui, X. (3) 575-586

Liang, J., see Hu, Y. (4) 863-868

Liang, Y., see Sun, X. (1) 123-134

Lin, X.-L., see Zhang, J. (1) 153-159

Lindberg, O., see Walterfang, M. (4) 897-906

Liu, J., see Sui, X. (3) 575-586

Liu, Q.-Y., see Tan, L. (4) 1017-1027

Liu, S., see Zhong, X. (4) 953-966

Liu, X., S. Wang, X. Zhang, Z. Wang, X. Tian and Y. He, Abnormal Amplitude of Low-Frequency Fluctuations of Intrinsic Brain Activity in Alzheimer's Disease (2) 387-397

Liu, X., Z. Wang, Y. Xia, G. Yu, K. Zeng, H. Luo, J. Hu, C.-X. Gong, J.-Z. Wang, X.-W. Zhou and X.C. Wang, Losartan-Induced Hypotension Leads to 
Tau Hyperphosphorylation and Memory Deficit (2) 419-427

Liu, Y., see Qiang, M. (4) 1039-1053

Lizio, R., see Cavedo, E. (4) 941-952

Lladó, A., see Balasa, M. (4) 919-927

Lleó, A., see Ramos-Fernández, E. (3) 643-657

Lobo, R.A., see Wharton, W. (2) 331-341

Londos, E., see Nielsen, H.M. (2) 343-350

Looi, J.C.L., see Walterfang, M. (4) 897-906

Lorenzo, P., see Bedse, G. (3) 701-712

Louis, E.D., see Romero, J.P. (2) 465-473

Love, S., see Miners, J.S. (3) 727-735

Lovestone, S., see Khan, W. (1) 37-43

Łuczak, M., see Suszyńska-Zajczyk, J. (3) 713-726

Luders, E., see Walterfang, M. (4) 897-906

Luo, H., see Liu, X. (2) 419-427

Luo, J., see Grammas, P. (3) 619-630

Luo, X., see Zhong, X. (4) 953-966

Lyutvinskiy, Y., see Yang, H. (3) 659-666

Ma, Q., see Sui, X. (3) 575-586

Macaulay, S.L., see Buckley, R.F. (3) 551-561

Macaulay, S.L., see Rembach, A. (1) 95-104

Maier, C.S., see Gray, N.E. (2) 359-373

Majoie, C.B., see Schmand, B. (2) 409-418

Maler, J.M., see Zimmermann, R. (4) 1055-1060

Maletta, R., see Piaceri, I. (3) 679-685

Maloney, B., see Perez, F.P. (1) 1-17

Maltsev, A.V., R. Santockyte, S. Bystryak and O.V. Galzitskaya, Activation of Neuronal Defense Mechanisms in Response to Pathogenic Factors Triggering Induction of Amyloidosis in Alzheimer's Disease (1) 19-32

Mandal, P.K., see Saharan, S. (3) 519-529

Manganiello, S., see Gharbiya, M. (4) 907-917

Manson, J.E., see Wharton, W. (2) 331-341

Marczak, Ł., see Suszyńska-Zajczyk, J. (3) 713-726

Mari, D., see Abbate, C. (4) 849-855

Mari, D., see Gussago, C. (1) 45-49

Marini, S., see Bracco, L. (3) 737-742

Marino, S., see Cavedo, E. (4) 941-952

Marra, C., see Minati, L. (1) 213-220

Martinez, J., see Grammas, P. (3) 619-630

Martini, C., see Pertl, M.-T. (3) 531-540

Martins, R.N., see Buckley, R.F. (3) 551-561

Martins, R.N., see Rembach, A. (1) 95-104

Maruff, P., see Buckley, R.F. (3) 551-561

Maruff, P., see Rembach, A. (1) 95-104

Masters, C.L., see Buckley, R.F. (3) 551-561

Masters, C.L., see Rembach, A. (1) 95-104

Mastropasqua, C., see Minati, L. (1) 213-220
Matsubara, S., see Homma, T. (1) 51-55

Mazat, L., see Delva, F. (1) 57-67

Meckler, X. and F. Checler, Visualization of Specific $\gamma$-Secretase Complexes using Bimolecular Fluorescence Complementation (1) 161-176

Medeiros, L.R., see Rosa, M.I. (2) 443-454

Meissner, C., see Gerschütz, A. (1) 135-142

Merriam, G., see Wharton, W. (2) 331-341

Miller, V.M., see Wharton, W. (2) 331-341

Minati, L., D. Chan, C. Mastropasqua, L. Serra, B. Spanò, C. Marra, C. Caltagirone, M. Cercignani and M. Bozzali, Widespread Alterations in Functional Brain Network Architecture in Amnestic Mild Cognitive Impairment (1) 213-220

Miners, J.S., R. Jones and S. Love, Differential Changes in $A \beta_{42}$ and $A \beta_{40}$ with Age (3) 727-735

Minthon, L., see Nielsen, H.M. (2) 343-350

Mitchell, A.J., see Kilimann, I. (3) 687-700

Mizutani, T., see Homma, T. (1) 51-55

Mochizuki, Y., see Homma, T. (1) 51-55

Mohades, S., see Wu, L. (4) 993-1004

Molinuevo, J.L., see Balasa, M. (4) 919-927

Molloy, D.W., see O’Caoimh, R. (3) 595-603

Monoranu, C.M., see Gerschütz, A. (1) 135-142

Montella, P., see Cavedo, E. (4) 941-952

Morley, J.E., see Farr, S.A. (4) 1005-1016

Morré, J., see Gray, N.E. (2) 359-373

Mouton-Liger, F., see Troussière, A.C. (4) 857-861

Mroczko, B., M. Groblewska, M. Zboch, A. Kulczyńska, O.M. Koper, M. Szmitkowski, J. Kornhuber and P. Lewczuk, Concentrations of Matrix Metalloproteinases and their Tissue Inhibitors in the Cerebrospinal Fluid of Patients with Alzheimer's Disease (2) 351-357

Mroczko, B., see Zimmermann, R. (4) 1055-1060

$\mathrm{Mu}, \mathrm{N}$., see Zhong, X. (4) 953-966

Muñoz, F.J., see Ramos-Fernández, E. (3) 643-657

Na, D.L., see Cho, H. (2) 285-295

Na, D.L., see Noh, Y. (4) 877-886

Nabrozidis, A., see García-Casares, N. (2) 375-386

Nacmias, B., see Piaceri, I. (3) 679-685

Naftolin, F., see Wharton, W. (2) 331-341

Nägga, K., see Nielsen, H.M. (2) 343-350

Najem, D., see Bamji-Mirza, M. (1) 105-122

Nannucci, S., see Piaceri, I. (3) 679-685

Neal-Perry, G., see Wharton, W. (2) 331-341

Nees, F., see Khan, W. (1) 37-43

Nelles, G., see Dias, I.H.K. (2) 399-408

Newhouse, S., see Khan, W. (1) 37-43

Nho, K., see Perez, F.P. (1) 1-17 
Ni, J., see Sui, X. (3) 575-586

Nicolini, P., see Abbate, C. (4) 849-855

Nicolini, P., see Gussago, C. (1) 45-49

Niehoff, M.L., see Farr, S.A. (4) 1005-1016

Nielsen, H.M., S. Hall, Y. Surova, K. Nägga, C. Nilsson, E. Londos, L. Minthon, O. Hansson and M. Wennström, Low Levels of Soluble NG2 in Cerebrospinal Fluid from Patients with Dementia with Lewy Bodies (2) 343-350

Nilsson, C., see Nielsen, H.M. (2) 343-350

Ning, Y., see Zhong, X. (4) 953-966

Noh, Y., S.W. Seo, S. Jeon, J.M. Lee, J.-H. Kim, G.H. Kim, H. Cho, C.W. Yoon, H.J. Kim, B.S. Ye, S.T. Kim, Y.S. Choe, K.-H. Lee, J.S. Kim, M. Ewers, M.W. Weiner, J.-H. Lee, D.J. Werring, D.R. Kang, C.S. Kim and D.L. Na, White Matter Hyperintensities are associated with Amyloid Burden in APOE4 Non-Carriers (4) 877-886

Noh, Y., see Cho, H. (2) 285-295

Nordin, L.E., see Walterfang, M. (4) 897-906

Nuñez, L., see Ramos-Fernández, E. (3) 643-657

O’Bryant, S.E., see Cunningham, R.L. (3) 563-573

O’Bryant, S.E., see Hall, J.R. (4) 887-896

O'Caoimh, R., L. Healy, Y. Gao, A. Svendrovski, D.M. Kerins, J. Eustace, P.G. Kehoe, G. Guyatt and D.W. Molloy, Effects of Centrally Acting Angiotensin Converting Enzyme Inhibitors on Functional Decline in Patients with Alzheimer's Disease (3) 595-603

Oberstein, T., see Zimmermann, R. (4) 1055-1060

Oliveira, C.R., see Rodrigues, D.I. (4) 981-992

Olives, J., see Balasa, M. (4) 919-927

Onwuteaka-Philipsen, B.D., see van der Steen, J.T. (3) 743-757

Osseby, G.-V., see Jacquin, A. (4) 1029-1038

Östberg, P., see Walterfang, M. (4) 897-906

Padiglioni, S., see Bracco, L. (3) 737-742

Paez, A., see Ramos-Fernández, E. (3) 643-657

Pallas-Bazarra, N., see Pérez, M. (1) 143-151

Palmer, R.F., see Royall, D.R. (3) 541-549

Palomer, E., see Ramos-Fernández, E. (3) 643-657

Paquet, C., see Troussière, A.C. (4) 857-861

Parisi, F., see Gharbiya, M. (4) 907-917

Park, N., see Janicki, S.C. (1) 83-93

Pasquier, F., see Troussière, A.C. (4) 857-861

Paus, T., see Khan, W. (1) 37-43

Pausova, Z., see Khan, W. (1) 37-43

Pepeu, G., see Bracco, L. (3) 737-742
Perez, F.P., D. Bose, B. Maloney, K. Nho, K. Shah and D.K. Lahiri, Late-Onset Alzheimer's Disease, Heating up and Foxed by Several Proteins: Pathomolecular Effects of the Aging Process (1) 1-17

Pérez, M., R. Cuadros, N. Pallas-Bazarra, C. García, E. Langa, J. Jurado-Arjona, F. Hernández and J. Avila, Boronate-Tau Mediated Uptake in Neurons (1) 143-151

Perry, G., see Rosen, A.C. (2) 233-235

Pertl, M.-T., T. Benke, L. Zamarian, C. Martini, T. Bodner, E. Karner and M. Delazer, Do Patients with Mild Cognitive Impairment Understand Numerical Health Information? (3) 531-540

Perucchi, J., see Rosa, M.I. (2) 443-454

Phillips, M., see Bayer, A. (1) 177-189

Piaceri, I., S. Pradella, C. Cupidi, S. Nannucci, C. Polito, S. Bagnoli, A. Tedde, N. Smirne, M. Anfossi, M. Gallo, L. Bernardi, R. Colao, R. Maletta, A.C. Bruni, S. Sorbi and B. Nacmias, Association of the Variant Cys139Arg at GRN Gene to the Clinical Spectrum of Frontotemporal Lobar Degeneration (3) 679-685

Piguet, O., see Savage, S.A. (2) 309-317

Pliássova, A., see Rodrigues, D.I. (4) 981-992

Poksay, K.S., see Zhang, Q. (3) 605-617

Polidori, M.C., see Dias, I.H.K. (2) 399-408

Polito, C., see Piaceri, I. (3) 679-685

Porter, G., see Bayer, A. (1) 177-189

Porter, V., see Ting, S.K.S. (2) 271-275

Pradella, S., see Piaceri, I. (3) 679-685

Puymirat, E., see Delva, F. (1) 57-67

Qiang, M., R. Xiao, T. Su, B.-B. Wu, Z.-Q. Tong, Y. Liu and R.-Q. He, A Novel Mechanism for Endogenous Formaldehyde Elevation in SAMP8 Mouse (4) 1039-1053

Quattrocchi, C.C., see Cavedo, E. (4) 941-952

Quinn, J.F., see Gray, N.E. (2) 359-373

Rainey-Smith, S.R., see Buckley, R.F. (3) 551-561

Rainfray, M., see Delva, F. (1) 57-67

Rajagopalan, P., see Walterfang, M. (4) 897-906

Rami, L., see Balasa, M. (4) 919-927

Ramos-Fernández, E., M. Tajes, E. Palomer, G. ILLRaga, M. Bosch-Morató, B. Guivernau, I. RománDégano, A. Eraso-Pichot, D. Alcolea, J. Fortea, L. Nuñez, A. Paez, F. Alameda, X. FernándezBusquets, A. Lleó, R. Elosúa, M. Boada, M.A. Valverde and F.J. Muñoz, Posttranslational 
Nitro-Glycative Modifications of Albumin in Alzheimer's Disease: Implications in Cytotoxicity and Amyloid- $\beta$ Peptide Aggregation (3) 643-657

Rauh, M., see Zimmermann, R. (4) 1055-1060

Reddy, P.H., Inhibitors of Mitochondrial Fission as a Therapeutic Strategy for Diseases with Oxidative Stress and Mitochondrial Dysfunction (2) 245-256 Redolfi, A., see Cavedo, E. (4) 941-952

Rembach, A., A.D. Watt, W.J. Wilson, V.L. Villemagne, S.C. Burnham, K.A. Ellis, P. Maruff, D. Ames, C.C. Rowe, S.L. Macaulay, A.I. Bush, R.N. Martins, C.L. Masters, J.D. Doecke and the AIBL Research Group, Plasma Amyloid- $\beta$ Levels are Significantly Associated with a Transition Toward Alzheimer's Disease as Measured by Cognitive Decline and Change in Neocortical Amyloid Burden (1) 95-104

Rembach, A., see Buckley, R.F. (3) 551-561

Ren, X., see Sui, X. (3) 575-586

Rice, A.C., P.M. Keeney, N.K. Algarzae, A.C. Ladd, R.R. Thomas and J.P. Bennett Jr., Mitochondrial DNA Copy Numbers in Pyramidal Neurons are Decreased and Mitochondrial Biogenesis Transcriptome Signaling is Disrupted in Alzheimer's Disease Hippocampi (2) 319-330

Richard, E., see Schmand, B. (2) 409-418

Riederer, P., see Gerschütz, A. (1) 135-142

Rienstra, A., see Schmand, B. (2) 409-418

Rietschel, M., see Khan, W. (1) 37-43

Riley, J., see Grammas, P. (3) 619-630

Ringman, J.M., see Ting, S.K.S. (2) 271-275

Rioja Villodres, J., see García-Casares, N. (2) 375-386

Robert, P., see Troussière, A.C. (4) 857-861

Robert, P.H., see Yakhia, M. (4) 869-875

Rodrigues, D.I., J. Gutierres, A. Pliássova, C.R. Oliveira, R.A. Cunha and P. Agostinho, Synaptic and Sub-Synaptic Localization of Amyloid-b Protein Precursor in the Rat Hippocampus (4) 981-992

Román-Dégano, I., see Ramos-Fernández, E. (3) 643657

Romano, A., see Bedse, G. (3) 701-712

Romano, A., see Feld, M. (1) 69-82

Romero, J.P., J. Benito-León, E.D. Louis and F. BermejoPareja, Alzheimer's Disease is Associated with Decreased Risk of Cancer-Specific Mortality: A Prospective Study (NEDICES) (2) 465-473

Rosa, M.I., J. Perucchi, L.R. Medeiros, B. Fernandes, M.E. Fernandes dos Reis and B.R. Silva, Accuracy of Cerebrospinal Fluid A $\beta_{1-42}$ for Alzheimer's
Disease Diagnosis: A Systematic Review and Meta-Analysis (2) 443-454

Rosa-Neto, P., see Wu, L. (4) 993-1004

Rosen, A.C., J.W. Ashford and G. Perry, Ethics Review as a Catalyst for Progress (2) 233-235

Rossi, P.D., see Abbate, C. (4) 849-855

Rossi, P.D., see Gussago, C. (1) 45-49

Rouaud, O., see Jacquin, A. (4) 1029-1038

Rowe, C.C., see Buckley, R.F. (3) 551-561

Rowe, C.C., see Rembach, A. (1) 95-104

Rowley, J., see Wu, L. (4) 993-1004

Royall, D.R. and R.F. Palmer, The Temporospatial Evolution of Neuritic Plaque-Related and Independent Tauopathies: Implications for Dementia Staging (3) 541-549

Rutishauser, D., see Yang, H. (3) 659-666

Sabatini, U., see Cavedo, E. (4) 941-952

Saharan, S. and P.K. Mandal, The Emerging Role of Glutathione in Alzheimer's Disease (3) 519-529

Saling, M.M., see Buckley, R.F. (3) 551-561

Salvatore, E., see Cavedo, E. (4) 941-952

Sanchez, A., see Grammas, P. (3) 619-630

Sánchez-Valle, R., see Balasa, M. (4) 919-927

Santockyte, R., see Maltsev, A.V. (1) 19-32

Santoro, M.N., see Wharton, W. (2) 331-341

Savage, G., see Buckley, R.F. (3) 551-561

Savage, S.A., O. Piguet and J.R. Hodges, Giving Words New Life: Generalization of Word Retraining Outcomes in Semantic Dementia (2) 309-317

Scarpini, E., see Abbate, C. (4) 849-855

Schamber, C., see Zimmermann, R. (4) 1055-1060

Scheff, S., see Abisambra, J.F. (3) 495-518

Schmand, B., A. Rienstra, H. Tamminga, E. Richard, W.A. van Gool, M.W.A. Caan and C.B. Majoie, Responsiveness of Magnetic Resonance Imaging and Neuropsychological Assessment in Memory Clinic Patients (2) 409-418

Schoemaker, D., see Wu, L. (4) 993-1004

Schrijvers, G., see Kraijo, H. (3) 631-642

Schultzberg, M., see Hanzel, C.E. (3) 667-678

Schumann, G., see Khan, W. (1) 37-43

Schupf, N., see Janicki, S.C. (1) 83-93

Scrascia, F., see Cavedo, E. (4) 941-952

Seo, S.W., see Cho, H. (2) 285-295

Seo, S.W., see Noh, Y. (4) 877-886

Seong, J.-K., see Cho, H. (2) 285-295

Serpente, M., see Abbate, C. (4) 849-855

Serra, L., see Minati, L. (1) 213-220

Severns, V., see Solberg, N.O. (1) 191-212 
Shah, K., see Perez, F.P. (1) 1-17

Shen, S., see Bamji-Mirza, M. (1) 105-122

Shen, Y., see Shi, Z. (2) 277-283

Shen, Z., see Zhong, X. (4) 953-966

Shi, H., see Zhong, X. (4) 953-966

Shi, S.-L., see Zhang, J. (1) 153-159

Shi, Z., Y. Wu, M. Wang, J. Cao, W. Feng, Y. Cheng, C. Li and Y. Shen, Greater Attenuation of Retinal Nerve Fiber Layer Thickness in Alzheimer's Disease Patients (2) 277-283

Shmuel, A., see Wu, L. (4) 993-1004

Sillerud, L.O., see Solberg, N.O. (1) 191-212

Silva, B.R., see Rosa, M.I. (2) 443-454

Silveri, M.C., see Boccia, M. (2) 455-463

Silverstein, Influence of Anesthetics on Alzheimer's

Disease: Biophysical, Animal Model, and Clinical Reports (4) 839-848

Simmons, A., see Khan, W. (1) 37-43

Sinforiani, E., see Cavedo, E. (4) 941-952

Singh, M., see Cunningham, R.L. (3) 563-573

Singh, M., see Hall, J.R. (4) 887-896

Smirne, N., see Piaceri, I. (3) 679-685

Smolka, M.N., see Khan, W. (1) 37-43

Soder, R.B., see Wu, L. (4) 993-1004

Soininen, H., see Yang, H. (3) 659-666

Solberg, N.O., R. Chamberlin, J.R. Vigil, L.M. Deck, J.E. Heidrich, D.C. Brown, C.I. Brady, T.A.V. Jagt, M. Garwood, M. Bisoffi, V. Severns, D.L.V. Jagt and L.O. Sillerud, Optical and SPION-Enhanced MR Imaging Shows that trans-Stilbene Inhibitors of NF- $\kappa \mathrm{B}$ Concomitantly Lower Alzheimer's Disease Plaque Formation and Microglial Activation in A $\beta$ PP/PS-1 Transgenic Mouse Brain (1) $191-212$

Sorbi, S., see Piaceri, I. (3) 679-685

Soricelli, A., see Cavedo, E. (4) 941-952

Soumyanath, A., see Gray, N.E. (2) 359-373

Spampinato, U., see Delva, F. (1) 57-67

Spanò, B., see Minati, L. (1) 213-220

Spilman, P., see Zhang, Q. (3) 605-617

Stahl, W., see Dias, I.H.K. (2) 399-408

Steenland, K., L. Zhao, F. Goldstein, J. Cellar, Biomarkers for Predicting Cognitive Decline in those with Normal Cognition (3) 587-594

Stevens, J.F., see Gray, N.E. (2) 359-373

Ströhle, A., see Khan, W. (1) 37-43

Su, T., see Qiang, M. (4) 1039-1053

Sui, X., X. Ren, P. Huang, S. Li, Q. Ma, M. Ying, J. Ni, J. Liu and X. Yang, Proteomic Analysis of Serum Proteins in Triple Transgenic Alzheimer's Disease Mice: Implications for Identifying Biomarkers for
Use to Screen Potential Candidate Therapeutic Drugs for Early Alzheimer's Disease (3) 575-586

Sun, X., Y. Liang, J. Wang, K. Chen, Y. Chen, X. Zhou, J. Jia and Z. Zhang, Early Frontal Structural and Functional Changes in Mild White Matter Lesions Relevant to Cognitive Decline (1) 123-134

Sun, Y., see Yang, Y. (4) 929-939

Surova, Y., see Nielsen, H.M. (2) 343-350

Suszyńska-Zajczyk, J., M. Łuczak, Ł. Marczak and H. Jakubowski, Hyperhomocysteinemia and Bleomycin Hydrolase Modulate the Expression of Mouse Brain Proteins Involved in Neurodegeneration (3) 713-726

Svendrovski, A., see O’Caoimh, R. (3) 595-603

Svensson, L., see Walterfang, M. (4) 897-906

Sziklas, V., see Wu, L. (4) 993-1004

Szmitkowski, M., see Mroczko, B. (2) 351-357

Szmitkowski, M., see Zimmermann, R. (4) 1055-1060

Szoeke, C., see Buckley, R.F. (3) 551-561

Tajes, M., see Ramos-Fernández, E. (3) 643-657

Takahashi, K., see Homma, T. (1) 51-55

Takahashi, M., see Homma, T. (1) 51-55

Takubo, H., see Homma, T. (1) 51-55

Tales, A., see Bayer, A. (1) 177-189

Tamminga, H., see Schmand, B. (2) 409-418

Tan, L., J.-T. Yu, M.-S. Tan, Q.-Y. Liu, H.-F. Wang, W. Zhang, T. Jiang and L. Tan, Genome-Wide Serum microRNA Expression Profiling Identifies Serum Biomarkers for Alzheimer's Disease (4) 1017 1027

Tan, L., see Tan, L. (4) 1017-1027

Tan, M.-S., see Tan, L. (4) 1017-1027

Tan, Y., see Zhong, X. (4) 953-966

Tatschner, T., see Gerschütz, A. (1) 135-142

Taylor, H., see Wharton, W. (2) 331-341

Tedde, A., see Piaceri, I. (3) 679-685

Tedeschi, G., see Cavedo, E. (4) 941-952

Tedone, E., see Gussago, C. (1) 45-49

Teipel, S.J., see Kilimann, I. (3) 687-700

Teunissen, C.E., see Del Campo (3) 481-494

Thangavel, R., see Xiong, Z. (2) 297-308

Thomas, R.R., see Rice, A.C. (2) 319-330

Thompson, P.M., see Walterfang, M. (4) 897-906

Tian, X., see Liu, X. (2) 387-397

Ting, S.K.S., T. Benzinger, V. Kepe, A. Fagan, G. Coppola, V. Porter, S. Hecimovic, S. Chakraverty, A.I. Alvarez-Retuerto, A. Goate and J.M. Ringman, A Novel PSEN1 Mutation (I238M) associated with Early-Onset Alzheimer's Disease in an African-American Woman (2) 271-275 
Tison, F., see Delva, F. (1) 57-67

Tong, Z.-Q., see Qiang, M. (4) 1039-1053

Torriglia, A., see Krantic, S. (2) 237-243

Trebbastoni, A., see Gharbiya, M. (4) 907-917

Tripathy, D., see Grammas, P. (3) 619-630

Troakes, C., see Gerschütz, A. (1) 135-142

Troussière, A.C., D. Wallon, F. Mouton-Liger, R. Yatimi, P. Robert, J. Hugon, D. Hannequin, F. Pasquier and C. Paquet, Who Needs Cerebrospinal Biomarkers? A National Survey in Clinical Practice (4) 857-861

Tu, S., see Flanagan, E.C. (1) 33-36

Uchihara, T., see Homma, T. (1) 51-55

Valverde, M.A., see Ramos-Fernández, E. (3) 643-657 Van den Block, L., see van der Steen, J.T. (3) 743-757 van der Flier, W.M., see Yakhia, M. (4) 869-875

van der Steen, J.T., M.C. van Soest-Poortvliet, M. Hallie-Heierman, B.D. Onwuteaka-Philipsen, L. Deliens, M.E. de Boer, L. Van den Block, N. van Uden, C.M.P.M. Hertogh and H.C.W. de Vet, Factors Associated with Initiation of Advance Care Planning in Dementia: A Systematic Review (3) 743-757

van Exel, J., see Kraijo, H. (3) 631-642

van Gool, W.A., see Schmand, B. (2) 409-418

van Soest-Poortvliet, M.C., see van der Steen, J.T. (3) 743-757

van Uden, N., see van der Steen, J.T. (3) 743-757

Velakoulis, D., see Walterfang, M. (4) 897-906

Vendemiale, G., see Bedse, G. (3) 701-712

Vernieri, F., see Cavedo, E. (4) 941-952

Vigil, J.R., see Solberg, N.O. (1) 191-212

Villemagne, V.L., see Rembach, A. (1) 95-104

Volbracht, C., see Dalby, N.O. (2) 429-442

Wagner, A.K., see Gerschütz, A. (1) 135-142

Wahlund, L.-O., see Walterfang, M. (4) 897-906

Wallon, D., see Troussière, A.C. (4) 857-861

Walterfang, M., E. Luders, J.C.L. Looi, P. Rajagopalan, D. Velakoulis, P.M. Thompson, O. Lindberg, P. Östberg, L.E. Nordin, L. Svensson and L.-O. Wahlund, Shape Analysis of the Corpus Callosum in Alzheimer's Disease and Frontotemporal Lobar Degeneration Subtypes (4) 897-906

Walton, J.R., Chronic Aluminum Intake Causes Alzheimer's Disease: Applying Sir Austin Bradford Hill's Causality Criteria (4) 765-838

Wang, H.-F., see Tan, L. (4) 1017-1027

Wang, J., see Sun, X. (1) 123-134
Wang, J.-Z., see Liu, X. (2) 419-427

Wang, M., see Shi, Z. (2) 277-283

Wang, S., see Liu, X. (2) 387-397

Wang, X.-C., see Liu, X. (2) 419-427

Wang, Z., see Liu, X. (2) 387-397

Wang, Z., see Liu, X. (2) 419-427

Watt, A.D., see Rembach, A. (1) 95-104

Weber, D., see Dias, I.H.K. (2) 399-408

Weiner, M.W., see Noh, Y. (4) 877-886

Wennström, M., see Nielsen, H.M. (2) 343-350

Werring, D.J., see Noh, Y. (4) 877-886

Westman, E., see Khan, W. (1) 37-43

Wharton, W., C.E. Gleason, N.M. Dowling, C.M. Carlsson, E.A. Brinton, M.N. Santoro, G. NealPerry, H. Taylor, F. Naftolin, R.A. Lobo, G. Merriam, J.E. Manson, M.I. Cedars, V.M. Miller, D.M. Black, M. Budoff, H.N. Hodis, S.M. Harman and S. Asthana, The KEEPS-Cognitive and Affective Study: Baseline Associations between Vascular Risk Factors and Cognition (2) 331-341

Wiechmann, A.R., see Hall, J.R. (4) 887-896

Wilson, W.J., see Rembach, A. (1) 95-104

Woodward, M., see Brodaty, H. (1) 221-226

Wu, B.-B., see Qiang, M. (4) 1039-1053

Wu, L., R.B. Soder, D. Schoemaker, F. Carbonnell, V. Sziklas, J. Rowley, S. Mohades, V. Fonov, P. Bellec, A. Dagher, A. Shmuel, J. Jia, S. Gauthier and P. Rosa-Neto, Resting State Executive Control Network Adaptations in Amnestic Mild Cognitive Impairment (4) 993-1004

Wu, R., see Zhong, X. (4) 953-966

Wu, Y., see Shi, Z. (2) 277-283

Xia, Y., see Liu, X. (2) 419-427

Xiao, R., see Qiang, M. (4) 1039-1053

Xiong, Z., R. Thangavel, D. Kempuraj, E. Yang, S. Zaheer and A. Zaheer, Alzheimer's Disease: Evidence for the Expression of Interleukin-33 and Its Receptor ST2 in the Brain (2) 297-308

$\mathrm{Xu}$, J., see Brodaty, H. (1) 221-226

Yakhia, M., A. König, W.M. van der Flier, L. Friedman, P.H. Robert and R. David, Actigraphic Motor Activity in Mild Cognitive Impairment Patients Carrying Out Short Functional Activity Tasks: Comparison between Mild Cognitive Impairment with and without Depressive Symptoms (4) 869875

Yang, E., see Xiong, Z. (2) 297-308

Yang, H., Y. Lyutvinskiy, S.-K. Herukka, H. Soininen, D. Rutishauser and R.A. Zubarev, Prognostic 
Polypeptide Blood Plasma Biomarkers of Alzheimer's Disease Progression (3) 659-666 Yang, X., see Sui, X. (3) 575-586

Yang, Y., S. Chen, J. Zhang, C. Li, Y. Sun, L. Zhang and $\mathrm{X}$. Zheng, Stimulation of Autophagy Prevents Amyloid-b Peptide-Induced Neuritic Degeneration in PC12 Cells (4) 929-939

Yang, Z., see Bamji-Mirza, M. (1) 105-122

Yatimi, R., see Troussière, A.C. (4) 857-861

Ye, B.S., see Cho, H. (2) 285-295

Ye, B.S., see Noh, Y. (4) 877-886

Yilmazer, S., see Gezen-Ak, D. (2) 257-269

Yin, X., see Grammas, P. (3) 619-630

Ying, M., see Sui, X. (3) 575-586

Yoon, C.W., see Cho, H. (2) 285-295

Yoon, C.W., see Noh, Y. (4) 877-886

Young, A., see Grammas, P. (3) 619-630

Yu, G., see Liu, X. (2) 419-427

Yu, J.-T., see Tan, L. (4) 1017-1027

Yu, S., see Hu, Y. (4) 863-868

Zaheer, A., see Xiong, Z. (2) 297-308

Zaheer, S., see Xiong, Z. (2) 297-308

Zamarian, L., see Pertl, M.-T. (3) 531-540

Zboch, M., see Mroczko, B. (2) 351-357

Zeng, K., see Liu, X. (2) 419-427

Zhang, C.-H., see Zhang, J. (1) 153-159

Zhang, H., see Zhong, X. (4) 953-966

Zhang, J., C.-H. Zhang, R.-J. Li, X.-L. Lin, Y.-D. Chen, H.-Q. Gao and S.-L. Shi, Accuracy of Urinary AD7c-NTP for Diagnosing Alzheimer's Disease:
A Systematic Review and Meta-Analysis (1) 153159

Zhang, J., see Yang, Y. (4) 929-939

Zhang, L., see Yang, Y. (4) 929-939

Zhang, Q., O. Descamps, M.J. Hart, K.S. Poksay, P. Spilman, D.J. Kane, O. Gorostiza, V. John and D.E. Bredesen, Paradoxical Effect of TrkA Inhibition in Alzheimer's Disease Models (3) 605-617

Zhang, W., see Bamji-Mirza, M. (1) 105-122

Zhang, W., see Tan, L. (4) 1017-1027

Zhang, X., see Liu, X. (2) 387-397

Zhang, Y., see Zhong, X. (4) 953-966

Zhang, Z., see Sun, X. (1) 123-134

Zhao, L., see Steenland, K. (3) 587-594

Zheng, D., see Zhong, X. (4) 953-966

Zheng, X., see Yang, Y. (4) 929-939

Zhong, X., H. Shi, Z. Shen, L. Hou, X. Luo, X. Chen, S. Liu, Y. Zhang, D. Zheng, Y. Tan, G. Huang, Y. Fang, H. Zhang, N. Mu, J. Chen, R. Wu and Y. Ning, ${ }^{1}$ H-Proton Magnetic Resonance Spectroscopy Differentiates Dementia with Lewy Bodies from Alzheimer's Disease (4) 953-966

Zhou, X., see Sun, X. (1) 123-134

Zhou, X.-W., see Liu, X. (2) 419-427

Zimmermann, R., E. Huber, C. Schamber, N. Lelental, B. Mroczko, S. Brandner, J.M. Maler, T. Oberstein, M. Szmitkowski, M. Rauh, J. Kornhuber and P. Lewczuk, Plasma Concentrations of the Amyloid-b Peptides in Young Volunteers: The Influence of the APOE Genotype (4) 1055-1060

Zubarev, R.A., see Yang, H. (3) 659-666 